\title{
LA TEORÍA DE LOS RECURSOS Y CAPACIDADES COMO FUNDAMENTO METODOLÓGICO PARA EL ESTUDIO DE LA GESTIÓN DE LA INNOVACIÓN EMPRESARIAL
}

\section{THE THEORY OF RESOURCES AND CAPABILITIES AS A METHODOLOGICAL FOUNDATION FOR THE STUDY OF MANAGEMENT OF BUSINESS INNOVATION}

Yureidys García Leonard, MSc. Máster en Administración de Negocios (Cuba). Docente de la Facultad de Turismo de la Universidad de La Habana, Cuba. ygarcia@ftur.uh.cu

Rafael Antonio Sorhegui Rodríguez, Lcdo. Licenciado en Contabilidad y Finanzas (Cuba). Docente de la Facultad de Ciencias Empresariales de la Universidad de La Habana, Cuba. rafael@fec.uh.cu

\section{ARTÍCULO DE REFLEXIÓN}

Recibido: 5 de noviembre de 2019.

Aceptado: 2 de diciembre de 2019.

\section{RESUMEN}

La innovación constituye en la actualidad una de las premisas fundamentales para el desempeño de las organizaciones inmersa en una economía global, caracterizada por un desarrollo exponencial de la ciencia y la técnica. Sin embargo, Latinoamérica es una de las regiones con mayor rezago en tema de innovación, como apunta los informes derivados del Índice Global de Innovación. Por tanto, instituciones como la CEPAL, la UNESCO y la RYCYT, destacan la necesidad de crear capacidades empresariales en la economía latinoamericana, como un elemento esencial para lograr una transformación productiva alejada del patrón de economías exportadoras de productos de bajo valor agregado. Razón por la cual potenciar la innovación en las empresas pasa por comprender el déficit de cultura innovadora que existe en el sector empresarial de la región. Bajo estas condiciones la teoría de recursos y capacidades se presenta como un referente metodológico viable para revertir la situación e inducir procesos asociados a la gestión de la innovación empresarial a través del desarrollo de capacidades dinámicas.

Palabras clave: teoría de recursos y capacidades, capacidades dinámicas, gestión de la innovación. 


\section{ABSTRACT}

Innovation is currently one of the fundamental premises for the performance of organizations immersed in a global economy, catheterized by an exponential development of science and technology. However, Latin America is one of the regions with the greatest lag in terms of innovation, as the reports derived from the Global Innovation Index point out. Therefore, institutions such as ECLAC, UNESCO and the RYCYT, highlight the need to build business capacities in Latin American economies, as an essential element to achieve a productive transformation away from the pattern of export economies of low value-added products. Reason for promoting innovation in companies involves understanding the deficit of innovative culture that exists in the business sector of the region. Under these conditions, the theory of resources and capabilities is presented as a viable methodological reference to reverse the situation and induce processes associated with the management of business innovation through the development of dynamic capabilities.

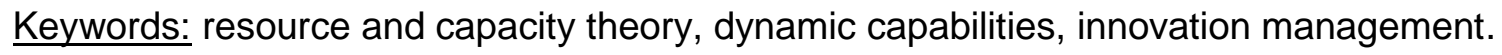

\section{INTRODUCCIÓN}

Innovar se ha convertido en una máxima para economías y empresas, de ahí que el estudio de la innovación es uno de los conceptos más difundidos. Un análisis bibliométrico realizado hasta el 2016, la innovación y el emprendimiento, junto al desarrollo sostenible son los temas de mayor cantidad de publicaciones en las revistas y en los sitios web de la ciencia, estas son provenientes de países como Chile, Estados Unidos de Norteamérica, España, Venezuela, México, Colombia, Reino Unido, Cuba y Brasil (Campo, Amar, y Olivero, 2018).

Albornoz (2009) definen a la innovación como un término polisémico, lo que de alguna manera promueve la existencia de abundantes definiciones acerca de lo que se entiende por innovación, no obstante es recurrente en los trabajos y artículos el concepto aportado por el manual OSLO (2005) "la introducción de un nuevo o significativo mejorado producto (bien o servicio), de un proceso, de un nuevo método de comercialización, o de un nuevo método organizativo, en las prácticas interna de la empresa, la organización en el lugar del trabajo o las relaciones exteriores", concepto que no toma distanciamiento de lo enunciado originalmente en la obra de Shumpeter (Shumpeter, 1939).

Con el establecimiento de la teoría evolucionista, el estudio de la innovación se ha deslindado en dos perspectivas, una donde predomina el análisis de los agentes que intervienen en el proceso de 
forma individual, cuyo foco es la empresa, y una segunda enfocado en la interrelación entre agentes que componen un sistema, en este marco es fundamental el trazado de políticas que permitan la creación de un espacio innovador.

A pesar de existir esta dicotomía metodológica para estudiar la innovación existe un punto de contacto donde se reconoce que innovar implica un esfuerzo superior a lo que puede una empresa de manera individual, existe consenso además que el logro de procesos que conduzcan a gestionar el conocimiento y el aprendizaje, son premisas para la innovación pues unido a las capacidades desarrolladas por la empresa permiten el impulso de actividades innovadoras conducente a valorizar los resultados de la organización Ahmed \& Sheperd (2012); Cotec (2010); Alburqueque, (1995), Beristain (2009); Bramuglia (2000); Bueno (2012); Dini \& Stumpo (2011); Escorsa \& Valls (2003), Herrera( 2011); Salinas (2012); Hidalgo (2011), estos autores reconocen que se necesita además promover la cooperación empresarial.

Autores como Lundval (2005, 2010), Dini \& Stumpo (2011), Arocena \& Sutz (2013), Lastres y Ávila (2013), Padillas, Gaudin y Rodríguez (2013), hacen énfasis en el carácter sistémico de la innovación y la relaciones entre diferentes agentes económicos; no obstante, aún desde esta perspectiva, la empresa ha sido el componente más estudiado.

Varios de los modelos que se desarrollan para lograr la innovación empresarial definen un entramado complejo de relaciones con agentes; no obstante, la mayoría de estos son más afines con las empresas poseedoras de una cultura innovadora y capacidades para tal fin. En tal sentido, referentes Metodológicos como es el caso del Manual de Oslo y Bogotá reflejan la necesidad que tienen las economías en desarrollo de fortalecer las capacidades para la innovación, siendo así la Teoría de los Recursos y Capacidades un marco de análisis fundamental en el estudio de la gestión de la innovación empresarial.

Reconocidas las carencias de las economías latinoamericanas y del caribe en el tema de construir capacidades empresariales para la innovación, en el presente artículo, se expone las ventajas que ofrece la Teoría de los Recursos y Capacidades para la gestión de la innovación; así como una descripción de las barreras fundamentales que cuenta las empresas del área para innovar.

\section{REVISIÓN TEÓRICA}

La teoría de los recursos y capacidades como perspectiva metodológica para analizar la gestión de la innovación empresarial. 
En la actualidad se reconoce la existencia de cincos generaciones de modelo como referentes para que las organizaciones realicen su proceso de innovación, partiendo de los llamados lineales que son actualmente poco operativos (Escorsa \& Valls, 2003) y (López, Blanco, y Guerra, 2009), hasta los modelos en redes que persiguen superar el enfoque lineal.

Velazco y Zaramillo (2008) y Barreto y Petit (2017) plantean que los modelos no captan toda la realidad innovadora, que se centran más en el proceso de desarrollo de productos y que no son muy prácticos para el sector de los servicios, que están orientados a empresas de gran tamaño pues se enfocan más en concebir una innovación de tipo radical que las incrementa. Alvares y Rosa (2010) plantean que resumir la complejidad del fenómeno en un esquema no capta lo diverso que puede ser el proceso.

Sin embargo, Velazco y Zaramillo (2008) reconocen la utilidad de los modelos y que estos deben centrarse en las características sectoriales y en los tipos de innovación. González y Martínez (2011) consideran que un aspecto distintivo de las investigaciones en innovación es la diversidad de enfoque adoptado atendiendo a la unidad de análisis seleccionada, estos criterios validan la existencia de múltiples modelos para la gestión de la innovación empresarial y no formas universales.

La anterior afirmación refuerza la tendencia a la creación de modelos específicos para gestionar la innovación por sectores y tipos de actividad; sin embargo, mucho de estos se centran en describir el proceso, basado en la definición de actividades para la innovación, adoleciendo de un adecuado tratamiento de cómo promover el desarrollo de las capacidades, al centrase más en la descripción del proceso atiendo en menor medida el proceso de ajuste empresarial en dependencias con las estructuras de recurso con los cuales cuenta la organización para innovar.

Al hablar del análisis de la gestión de la innovación en empresa una de las disciplinas que más aporta es la dirección González y Martínez, (2011), recientemente los estudios más contemporáneos en esta área utilizan como fundamento la teoría de las capacidades y recursos y la compresión del papel esencial que juegan los intangibles en la rentabilidad empresarial.

Para Garzón (2015) el enfoque de los recursos y capacidades surge desde la dirección estratégica y por la necesidad de explicar la diferencia de resultados entre organizaciones, define al enfoque como integrador al nutrirse de otras teorías. Continuando con la lógica expositiva de Garzón, los basamentos originarios del citado enfoque se encuentran en la teoría evolucionista ambos 
paradigmas metodológicos coinciden con el papel que juega la heterogeneidad de las organizaciones ante la diferencia de la productividad entre sectores.

La lógica que imprime el evolucionismo para estudiar a la empresa radica en la importancia que se le confiere a los recursos con que cuenta la organización como condicionante del desempeño, de ahí la existencia de comportamientos diferentes entre empresa, que aún en condiciones similares de competencia y estructura de mercado, existen desniveles en la eficacia y rendimiento de las inversiones, los resultados provenientes de la I+D+i, muestran también una disparidad por sectores de la economía.

En este marco cobra interés el análisis estratégico más centrado en el ámbito interno de la empresa , siendo esto una consecuencia de la consolidación de la Teoría de los recursos y Capacidades (TRC), Valencia y Rodríguez (2015) apuntan que la esencia de la TRC es identificar el potencial de recursos disponibles que posee y controla la organización, determinar las capacidades a través de como las habilidades de sus miembros pueden integrar, construir y reconfigurar su dotación de recursos, lo cual indudablemente modifica sus capacidades iniciales, todo esto para crear nuevos productos y procesos.

Valencia y Rodríguez (2015) acotan que para la existencia de innovación es necesaria una buena combinación de capacidades, habilidades y recursos, de ahí que la TRC es el marco idóneo para desplegar el estudio de la gestión de la innovación empresarial, el establecimiento de capacidades conduce al desarrollo de nuevas habilidades y destreza con una impacto en el desempeño innovador de las empresas, al estar constantemente la organización en un proceso de creación de capacidades demanda por la inestabilidad del entorno en que se desenvuelven las empresas.

Una categoría esencial dentro de la TRCE lo constituye las capacidades, no obstante en la literatura revisada constituye un término donde no existe un consenso, incluso se observa el empleo indistinto de términos como capacidades, habilidades y rutinas para describir un mismo fenómeno en la organización Cruz, Navas, \& López (s/a) y Garzón (2015), argumentan que la amplia gama de acepciones, así como la diversidad de definiciones proviene de la coexistencia de tres enfoques para definir las capacidades.

Un enfoque orientado a la innovación o sea la construcción de capacidades para innovar, a través de la absorción del conocimiento y el aprendizaje, un segundo asociado a la construcción de capacidades y en este constructo en donde se encuentra la mayoría de las definiciones, la esencia estriba en entenderlas como una combinación de recursos de la empresa para adaptarse al entorno, 
y un tercer enfoque denominado contingente donde se conciben a las capacidades como mecanismos de ajustes ante los cambios en el entorno.

Se considera acertado el planteamiento de Cruz, Navas, y López (s.f.) sobre la no excluencia de los enfoques, bajo el paradigma metodológico de la TRC, estos se auto-refuerzan, Cruz, Navas, y López (s.f.) la existencia de tres enfoque no limitan que en las definiciones existente se comportan criterios como: la capacidad de adaptación que debe desarrollar la empresa y el reconocimiento de la influencia del entorno inestable sobre la organización.

Se puede afirmar entonces que la esencia de los tres enfoques se encuentran sustentados en el seno de la TRC que identifica a la empresa en un constante proceso de desarrollo, ajuste y adaptación, debido a la influencia del entorno competitivo, y la propia estructura del mercado, así como tendencias de variables externas que rebasan los marcos de la competencia empresarial, por lo que continuamente se encuentran enfrentando oportunidades y problemas que deben resolver a partir de los recursos con que cuentan y las habilidades de sus miembros, la confluencia del ajuste de recurso de la organización por el accionar de sus miembros promueve el desarrollo de capacidades.

Siendo así Dávila (2013) define a las capacidades organizacionales como un proceso de construcción social para solucionar problemas, las cuales evolucionan con el tiempo mediante un proceso de aprendizaje, ligando el concepto a la acción y al desempeño.

Vincular a la capacidad con la acción y con el ajuste de la organización con el entorno implica que como categoría (capacidad) es dinámica, por lo que en la literatura se emplea el término capacidades dinámicas. Yoguel, Barletta, y Pereira (2013) consideran que el enfoque de capacidades dinámicas es consistente con la idea schumpeteriana de que las firmas buscan constantemente crear nuevas combinaciones y las empresas rivales intentan desarrollar nuevas competencias o mejorar las que hay para imitar las nuevas combinaciones. Para ello, resultan claves tanto la construcción de capacidades como los cambios organizacionales (Yoguel, Barletta, \& Pereira, 2013).

Cruz, Navas, y López (s.f.) definen el concepto de capacidades dinámicas como la habilidad de la empresa de generar nuevas capacidades organizativas a partir de la adquisición o el desarrollo de nuevos recursos y/o la recombinación de forma novedosa de los ya existentes, lo que resulta especialmente relevante ante regímenes externos caracterizados por rápidos cambios (Cruz, Navas, y López , s.f.). 
(Teece, 2018) Las considera dinámica porque a partir de las establecidas, las organizaciones necesitan crear nuevas capacidades de cara al futuro de la empresa, para Miranda (2015) las considera esenciales para el logro de una ventaja competitiva sostenible y Aguilar y Yepes (2006), como la base de la reconfiguración de los recursos de la organización.

Finalmente, Garzón (2015) basado en una revisión bibliográfica que el realiza propone la siguiente definición de capacidades dinámicas: potencialidad (inimitable) de la empresa para generar nuevos saberes organizacionales a partir de una continua creación, ampliación, mejoramiento, protección, integración, reconfiguración, renovación, recreación, incremento y reconstrucción de sus core competences, para poder responder a los cambios en mercados y tecnologías, que incluyen la habilidad de la empresa de configurar el entorno en el que opera, desarrollando nuevos productos y/o procesos y diseñando e implementando nuevos o redefinidos modelos de negocio plausibles para conseguir mantener y/o ampliar las ventajas competitivas.

La anterior definición nos conduce a lo que Dávila (2013) identifica, a la capacidad como un patrón y el cual no puede ser denominado como tal hasta que no demuestre su efectividad ante variadas situaciones, al constituirse la capacidad como un patrón establece el carácter dual de esta que por naturaleza es dinámica y por esencia es rutinaria.

Aguilar (2005), Aguilar y Yepes (2006), Benavides y Quintana (2006), Lovera, Castro y otros (2008), colocan a las rutinas como la esencia evolutiva de las empresas, al ser modelos de acción recurrentes y comportamentales que brindan soluciones a problemas. Constituyen procedimientos asentados en la cultura de la empresa que resultan en formas de hacer las cosas (Pulido, s.f.), Aguilar (2005) resalta que la importancia estriba en que complementan vacíos que las reglas y procedimientos estándar estipulan.

Yoguel, Barletta, y Pereira (2013) que consideran que con la teoría evolutiva se retoma el núcleo duro de la teoría de Shumpeter estableciendo la conexión entre innovación, rutinas y capacidades dinámicas, esta línea la desarrolla Dávila (2013) quien plantea que la corriente del pensamiento evolutiva establece a la firma como una jerarquía de rutinas que evolucionan hacia capacidades dinámicas, varias son las críticas que se le hacen a la escuela evolutiva acerca de que si las rutinas constituyen o no un freno para la innovación y el cambio.

Lo cierto es que la innovación se asocia al cambio y a la creación, por lo que el proceso conduce a la rigidez empresarial podría ser contraproducente; sin embargo, en la práctica innovar depende 
además de la creatividad, la existencia de un pensamiento lógico, organizado estructurado que permita desarrollar las ideas, si bien es cierto que a los modelos se le ha criticado su carácter secuencial para el desarrollo de productos, procesos y cambios organizacionales, se precisa que las capacidades de innovación se conviertan en rutina y marque una cultura organizacional.

La capacidad de innovación se configura como un elemento clave para la obtención de resultados empresariales a través de los procesos de explotación y exploración organizacional (Prado, 2013).

Valencia y Rodríguez (2015) la entienden como las habilidades que las personas desarrollan para lograr nuevas combinaciones de los elementos internos y externos de la organización, Aguilar y Yepes (2006) la ven como las capacidades que pueda desarrollar procesos de trasformación, Garzón (2015) lo asocia más hacia la gestión del conocimiento.

En apretada síntesis las capacidades de innovación apuntan a la habilidad de la empresa para recombinar recursos, crear, mejorar y adaptarse, en el marco de economías en vías de desarrollo donde su naturaleza es la asimilación de tecnología, es necesario incluir como capacidades de innovación: capacidades de adquisición y asimilación tecnológica, esto supone un proceso de aprendizaje para incorporar la tecnología en los procesos internos de la organización, lo que inexorablemente lleva a que forme parte de las capacidades de innovación de este grupo de países, el aprendizaje y la gestión del conocimiento.

\section{El desarrollo de las capacidades y la gestión de la innovación empresarial en Latinoamérica}

El bajo nivel de las capacidades de innovación que tienen las economías de Latinoamérica es reconocido por La Red Iberoamericana/Interamericana de Indicadores de Ciencia y Tecnología (RICYT), que de conjunto con COLCIENCIA diseñó el Manual de Bogotá el cual se encamina más al análisis de la gestión de las actividades de innovación, así como los esfuerzos empresariales por asimilar conocimiento y tecnología foránea, por lo que los temas de capacitación y la vigilancia tecnológica, son ejes prioritarios para las economías en desarrollo (Bogotá, 2001).

(Castellano, 2019) Considera que los países de América Latina están quedándose en la periferia de la Cuarta Revolución Industrial. El bajo crecimiento económico latinoamericano una larga desaceleración que se remonta a 2013 y la ausencia de reformas estructurales condenan a la región a cumplir un papel secundario en ese contexto internacional. Datos que demuestran está realidad en el continente provienen de los resultados de Índice Global de Innovación donde solo Chile, Costa Rica y México fueron las naciones latinoamericanas mejor posicionadas (ONU, 2019). 
Para (Castellano, 2019) los grandes déficits regionales en materia de innovación, responde a la inversión América. La figura 1 muestra el por ciento que representa el gasto de I+D en el Producto Interno Bruto (PIB) de América Latina y el Caribe, el cual muestra una tendencia inestable, se le suma además su baja contribución al PIB regional al tomar valores por debajo del 1 por ciento del total del (PIB) regional.

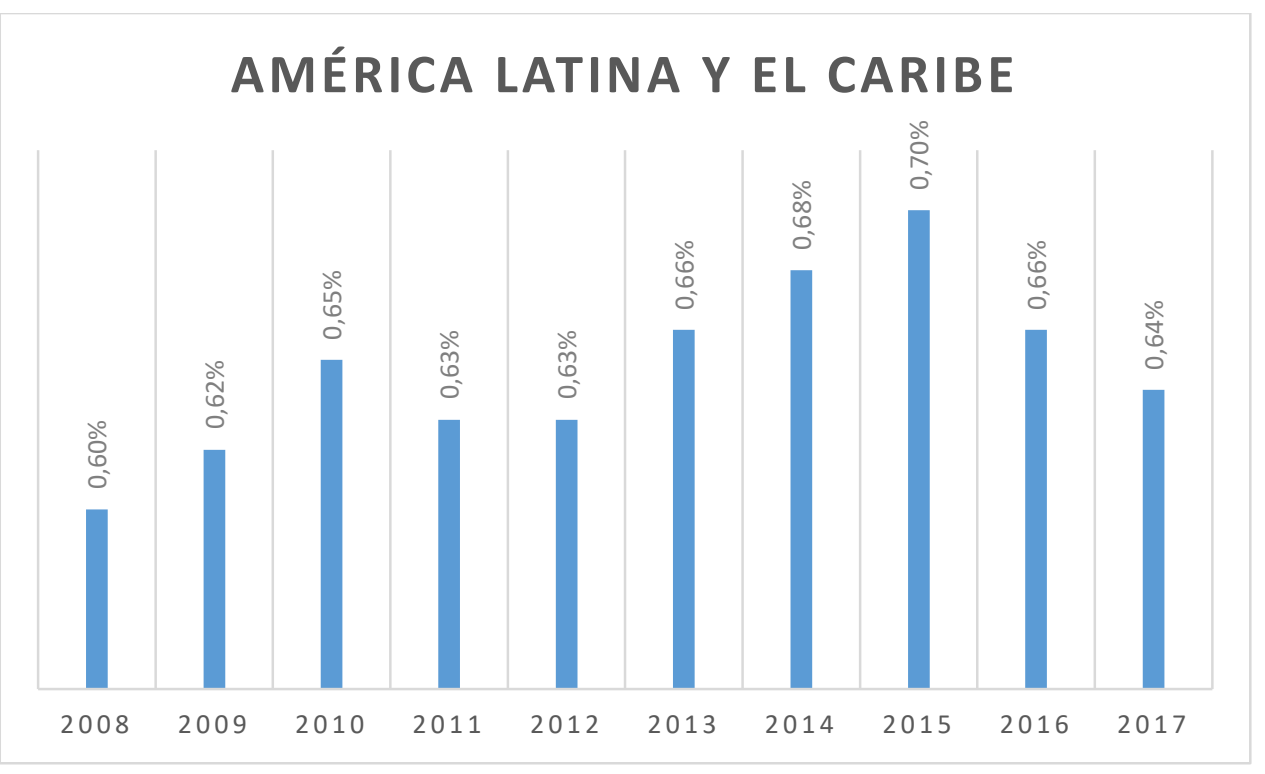

Figura 1. Por ciento del Gasto en I+D con relación al PIB.

Fuente: Elaboración propia a partir de datos de la RYCYT.

La CEPAL reconoce la existencia en la región de un rezago en términos de estructura productiva, y de capacidades tecnológicas; así como la baja propensión de los países latinoamericanos a invertir en I+D, con excepción del Brasil, la Argentina, Costa Rica y México (CEPAL, 2016), sumado a lo que (Castellano, 2019) plantea como una estructura económica que no se basa en sectores tecnológicamente avanzados, sino en una competitividad basada en costes menores. Con una productividad laboral decreciente desde los 70 , son dos componentes que sin lugar a duda constituyen un obstáculo al desarrollo de capacidades para la innovación.

Se incorpora como característica del área que las principales inversiones en materia de $1+D$, provienen del sector público aspecto que dista de la realidad de las economías innovadoras donde el sector privado tiene una presencia importante en el aporte de fondos para la innovación, este hecho en sí corrobora la tesis de Castellano en cuanto a la apatía de los empresarios en invertir en $I+D$, ante la presencia de los riesgo asociado a la innovación y los déficit en las políticas de innovación de proteger los resultados, para él la esencia está en que el sector privado 
latinoamericano sigue enclaustrado en el retraimiento a la hora de invertir en innovación por razones históricas y de cultura (Castellano, 2019).

El contexto latinoamericano trasluce la necesidad de cambios en la base productiva y fortalecimiento de los Sistemas de Innovación Nacional (SIN), el fortalecimiento de los diferentes actores que intervienen en el sistema, sin embargo, estudios realizados por la CEPAL reconoce que en el área, los sistemas educativos, componente fundamental de los (SIN) son débiles en cuanto al desarrollo de personal dedicado a la I+D, debido a que la estructura productiva no demanda un personal con capacidades para la innovación (CEPAL, 2016), dicha afirmación implica el reconocimiento de la debilidad de los Sistemas Nacionales de Innovación en la región que refuerzan el problema del desarrollo de capacidades empresariales para innovar, problema que repercute en la gestión de la innovación empresarial componente central del SIN.

Ahmed \& Sheperd (2012) definen que la gestión de la innovación en empresa pasa por determinar cuáles son los conocimientos y capacidades de una organización para su supervivencia a largo plazo.

Siendo así se retoma el punto de partida la importancia de las capacidades empresariales como el fundamento esencial para modelar el performance empresarial en un entorno donde el desarrollo acelerado de la Técnicas de la Información y la Comunicación, ponen en tela de juicio los modelos convencionales de gestión empresarial hipotecando así las estructuras productivas de los países latinoamericanos y reproduciendo el patrón de especialización productiva muy vinculado a las llamadas ventajas comparativas estáticas que se caracteriza por la exportación de bienes intensivos en trabajo, con bajos salarios y una fuerte presencia de empresas maquiladoras (Lemarchand, 2010)

De ahí que los modelos rígidos de dirección empresarial son inoperantes en la actualidad, la flexibilidad y el cambio son palabras de orden en la gestión empresarial, en el comercio internacional es clave las producciones de valor agregado, surgen servicios intensivos en conocimientos, impactan tendencias económicas a nivel empresarial como: el internet de las cosas, la multifuncionalidad de las aplicaciones en dispositivos móviles, inteligencia artificial, todas estas modificando las instituciones.

Bajo estas condiciones compiten las empresas del área geográfica, de ahí que el desarrollo de la innovación se convierte en un aspecto de supervivencia de cara al futuro, fortalecer las capacidades que permitan ya no solo crear, sino asimilar los cambios y las tendencias de la economía es una 
cuestión de primer orden.

Como alternativa para lograr una reducción de la brecha en materia de innovación de las empresas del área, Lemarchand propone objetivos que debe perseguir la política tecnológica e innovación:

a) Apoyo para la formación de recursos humanos en la empresa;

b) Apoyo al desarrollo de capacidades productivas de la empresa;

c) Fortalecimiento de las capacidades públicas de C y T destinadas a la innovación;

d) Impulso para la creación de empresas de base tecnológica;

e) Promoción de la cooperación público/privada;

f) Protección de la propiedad industrial;

g) Políticas de innovación vertical;

h) Políticas de innovación horizontal;

i) Políticas de innovación selectiva. (Lemarchand, 2010)

\section{CONCLUSIONES}

La corriente del pensamiento evolucionista continúa siendo un paradigma de estudio esencial para el análisis de la innovación, la propuesta que ofrece la teoría de los recursos y capacidades en el estudio de la estrategia empresarial continúa la línea del evolucionismo de entender a la empresa como una jerarquía de rutina.

Las realidades de las economías latinoamericanas en cuanto a los temas de innovación apuntan a que el fortalecimiento de las capacidades empresarial sea uno de los objetivos esenciales a promover y desarrollar en la gestión de la innovación empresarial, y en este marco la teoría de los recursos y capacidades brindan un marco metodológico para el análisis y trazado de estrategia de innovación.

Estudios realizados por organizaciones como la CEPAL y la UNESCO demuestra que el sistema productivo de Latinoamérica presenta rezagos con respecto a los cambios en las estructuras económicas de los países, por lo que se precisa de trabajar en dos ejes esenciales fortalecer los (SIN) y desarrollar capacidades empresariales.

\section{REFERENCIAS BIBLIOGRÁFICAS}

Aguliar, J., y Yepes, H. (2006). Gestión de capacidades dinámicas e innovación: Una aproximación 
conceptual. Ciencias Administrativa, 1-15.

Ahmed, P., y Sheperd, C. (2012). Administración de la innovación. México D.F.: Pearson

Albornoz, M. (2009). Indicadores de innovación: las dificultades de un concepto en evolución.

Revista iberoamericana de ciencia, tecnología y sociedad, 9-25.

Alburqueque, F. (Junio de 2013). Economía del desarrollo y desarrollo territorial. Consultado el

15 de mayo de 2017, Recuperado de

https://dialnet.unirioja.es/servlet/articulo?codigo=2576588

Arocena, R., y Sutz, J. (2013). Innovación y democratización del conocimiento. En G. D. Sutz, Sistemas de innovación para un desarrollo inclusivo. La experiencia latinoamericana (pp. 1-347). Dsitrito Federal: LALICS. Recuperado de: eva.fcs.edu.uy > mod_folder > content > u4 - Arocena-Sutz 2003

Arocena, R., y Sutz, J. (2013). Navegando contra el viento Ciencia, Tecnología y

Subdesarrollo.

Barreto , J., y Petit, E. (2017). Modelos explicativos del proceso de innovación tecnológica en las organizaciones. Revista Venozolana de Gerencia, 22-79.

Benavides Velasco, C., y Quintana García, C. (2006). Inteligencia competitiva prospectiva e innovación La norma UNE-166006 EX sobre el sistema de vigilancia tecnológica. Boletín Económico, 47-63.

Beristain, L. M. (2009). Una Revisión de la Interpretación Económica sobre la Innovación. Journal of Technology Management \& Innovation. Universidad Alberto Hurtado, Facultad de Economía y Negocios, 140-150.

Bramuglia, C. (2000). La tecnología y la Teoría Económica de la innovación. Consultado el 5 de mayo de 2016. Recuperado de Google acádemico: http://www.fsoc.uba.ar

Bueno, E. (2012). El capital intelectual como sistema generador de emprendimiento e innovación. Consultado el 27 de noviembre de 2016, Recuperado de:

http://www.minetur.gob.es/Publicaciones/Publicacionesperiodicas/Economialndustrial/Revis taEconomialndustrial/388/Eduardo\%20Bueno.pdf

Campo, L., Amar, P., y Olivero, E. (2018). Emprendimiento e innovación como motor del desarrollo sostenible: Estudio bibliométrico (2006-2016). Revista Ciencias Sociales, 26-37.

Castellano, R. N. (11 de septiembre de 2019). ¿Por qué no prospera la innovación en América Latina? Política, economía e ideas sobre el mundo en español. Recuperado de: https://www.esglobal.org/por-que-no-prospera-la-innovacion-en-america-latina/

CEPAL. (25 de Noviembre de 2019). Ciencia, tecnología e Innovación en la economía digital. La situación de América Latina y el Caribe. Cepal, Recuperado de: 
https://innovalac.cepal.org/2/es/documentos/ciencia-tecnologia-e-innovacion-la-economiadigital-la-situacion-america-latina-caribe.html

COTEC. (2010). La innovación en sentido amplio:un modelo empresarial. Análisis conceptual y empírico. Madrid: COTEC.

Cruz, J., Navas, J., y López, P. (s.f.). Concepto e implicaciones de las capacidades dinámicas desde un enfoque de dirección del conocimiento. Recuperado de http://www.aeca1.org/pub/on_line/comunicaciones_xvcongresoaeca/cd/64c.pdf

Dávila, J. C. (2013). Capacidades organizacionales: dinámicas por naturaleza. Cuadernos de Administración, 11-33.

Dini, M., y Stumpo, G. (2011). Políticas para la innovación en las pequeñas y medianas empresas en América Latina. Santiago de Chile.: CEPAL.

Escorsa, P., y Valls, J. (2003). Tecnología e Innovación en la empresa. Madrid: UPC.

Garzón, M.A. (2015). Modelo de capacidades dinámicas. Revista Dimensión Empresarial, Vol. 13, Núm. 1, p. 111-131.

González, C. y Martínez, J.L. (2014). Gerencia estratégica e innovación empresarial: referentes conceptuales. Revista Dimensión Empresarial, Vol. 12, Núm. 1, p. 107-116.

Herrera, R. (2011). Conocimiento, innovación y desarrollo. En R. H. González, Conocimiento, innovación y desarrollo (p. 290). Costa Rica, San José: I.G.E.

Hidalgo Nuchera, A. (2011). La gestión de la innovación como proceso. En R. H. González, Conocimiento, innovación y desarrollo (p. 290). San José: I.G.E.

Schumpeter, J. (1939). Business Cycles. Nueva York: McGraw-Hill, Vol. I, pp. 84 y 87-88.

Lastres, H. M., y otros. (2013). Innovación, sistemas de producción e innovación y la contribución del Banco de Desarrollo de Brasil (BNDES). En O. C. Dutrénit, Políticas de ciencia tecnología e innovación para el desarrollo: La experiencia latinoamericana ( $p$. 290). Distrito Federal: FCCyT.

Lemarchand, G. A. (2010). Las políticas de ciencia, tecnología e innovación en América 
Latina y el Caribe durante las últimas seis décadas. En G. A. Lemarchand, Sistemas nacionales de ciencia, tecnología e innovación en América Latina y el Caribe (p. 324). Montevideo: UNESCO.

López, O., Blanco, M., \& Guerra, S. (2009). Evolución de los modelos de la gestión de innovación. Innovaciones de Negocios, 251-264.

Lovera, M. I., Castro, E., y Smith, H. (2008). Evolucionismo económico desde la perspectiva de Nelson y Winter. Multiciencia, 48-54.

Lundvall , B.Å., Edquist, C., y Johnson, B. (2003). Economic Development and the National System of Innovation Approach . First Globelics Conference. Rio de Janeiro .

Miranda, J. (2015). El Modelo de las Capacidades Dinámicas en las Organizaciones. Investigación administrativa, 44(116). Consultado el 25 de noviembre de 2019. Recuperado de: http://www.scielo.org.mx/scielo.php?script=sci_arttext\&pid=S2448$76782015000200005 \&$ Ing=es\&tlng=es.

OCDE. (2005). Manual de Oslo. Recuperado de:

http://www.oecd.org/centrodemexico/publicaciones/

ONU. (24 de julio de 2019). Asuntos Económicos. Noticias ONU. Recuperado de: https://news.un.org/es/story/2019/07/1459671

Padillas, R., Gaudin, Y., y Rodríguez, P. (2013). Sistemas de Innovación. En R. P. Pérez, Sistemas de Innovación en Cenntro América. Fortalecimento a través de la integración regional (p. 963). Santiago de Chile: CEPAL.

Prado, J. C. (2013). Condiciones de la gestión del conocimiento, capacidad de innovación y resultados empresariales. Un modelo explicativo. Pensamiento y Gestión, 25-63.

RICYT. (2001). Manual de Bogotá. Normalización de indicadores de innovación tecnológica en América Latina y el Caribe. Recuperado de http://www.ricyt.org/component/docman/cat_view/16-manuales?ltemid=2

Riveros, B. P. (25 de junio de 2010). Teoría de los recursos y capacidades el foco estratégico centrado en el interior de la organización. Recuperado de Dialnet: https://dialnet.unirioja.es/servlet/articulo?codigo=5137577 
Robledo, J., López, C., y Zapata , W. (2010). Desarrollo de una Metodología de Evaluación de Capacidades de Innovación. Perfil de Coyuntura Económica, 133-148.

Salinas, M. I. (2012). Metodología para implemertar la gestión de lainnovación. Recuperado de: http://repositorio.uchile.cl/handle/2250/111361

Sánchez, M. A. (2009). Teoría económica evolutiva de la empresa: ¿una alternativa a la teoría neoclásica? Problemas del Desarrollo. Revista Latinoamérica de Economía, 163-183.

Teece, D. J. (5 de Mayo de 2018). Bussines models and dynamic capabilities. Recuperado de ELSEVIER: http://www.elsevier.com/locate//rp

Valencia, M. (2015). Capacidades dinámicas, innovación de producto e aprendizaje organizacional en pymes del sector cárnico. Ingeniería Industrial, 36(3), 287-305. Consultado el 26 de noviembre de 2019. Recuperado de:

http://scielo.sld.cu/scielo.php?script=sci_arttext\&pid=S1815$59362015000300007 \&$ Ing=es\&tlng=es.

Velazco, E., y Zamanillo, I. (2008). Evolución de las propuestas sobre el proceso de innovación: Qué se puede concluir de su estudio. Investigaciones Europeas de Dirección e Economía de Empresa, 127-138.

Yoguel, G., Barletta, F., y Pereira, M. (2013). De Schumpeter a los postschumpeterianos: viejas y nuevas dimensiones analíticas. Revista Problemas del Desarrollo, 35-59.

Zambrano, J. J. (2005). Modelos de acción organizacionales en la construcción de capacidades empresariales. Cuadernos de Administración, Vol. 18, Núm. 29, 87-102. 